\title{
NUEVOS DESARROLLOS EN EL TRATAMIENTO MÉDICO DEL GLAUCOMA
}

\author{
NEW DEVELOPMENTS IN GLAUCOMA MEDICAL TREATMENT
}

\author{
MUÑOZ-NEGRETE FJ ${ }^{1}$, PÉREZ-LÓPEZ M², WON KIM H-R ${ }^{2}$, REBOLLEDA G ${ }^{1}$
}

\begin{abstract}
RESUMEN
El tratamiento médico del glaucoma ha presentado una gran evolución en los últimos años. Las investigaciones que se están desarrollando en este campo se basan en el perfeccionamiento de productos activos preexistentes y en el descubrimiento de nuevos principios activos. En productos activos comercializados se busca obtener una mejor tolerancia local, utilizando conservantes menos tóxicos, como es el caso del preservante sofZia que se ha asociado al travoprost, y la eliminación del uso de conservantes, como sucede con el tafluprost. También el desarrollo de nuevas combinaciones fijas de fármacos comercializados podría facilitar su administración y el cumplimiento terapéutico.

Por otro lado, se están investigando nuevas moléculas que puedan ser de utilidad en el tratamiento médico del glaucoma. Entre estos podemos destacar las investigaciones sobre antagonistas del calcio como la lomerizina, que parece no producir hipotensión sistémica, y productos que se están investigando para uso tópico ocular como la flunarizina y la iganidipina. También se están investigando inhibidores de la endotelina 1, como el sulfisoxazol y la bunazosina. Dentro del sistema renina-angiotensina se están evaluando la Angiotensina (1-7) y el olmesartan. La investigación de drogas trabeculares, como los inhibidores de la Rho-kinasa, podrían actuar sobre el mecanismo patogénico del glauco-
\end{abstract}

\begin{abstract}
The medical treatment of glaucoma has undergone significant development in recent years. Research in this field is focused on improving pre-existing drugs and on the development of new molecules. In relation to commercial drugs, there is a trend to improve local tolerance, using less toxic preservatives as in the case of sofZIA in travoprost, and eliminating the preservatives as in tafluprost. The development of new, fixed combinations of commercial drugs could also enhance their administration and therapeutic compliance.

There is also intense research activity in the search for new therapeutic groups for glaucoma treatment. Calcium channel-blockers such as lomerizine do not seem to affect systemic hypotension, while topical calcium-blockers like flunarizine and iganidipine are also under research. Endothelin 1 antagonists such as sulfisoxazole and bunazosine could be also useful in the treatment of glaucoma. In the renin angiotensin system, angiotensin (1-7) and olmesartan are under investigation for use in glaucoma patients. Trabecular drugs such as Rho-kinase inhibitors could be effective on the pathogenic mechanism of primary open angle glaucoma.

Finally, topical mifepristone, an antagonist of glucocorticoid receptors, is under evaluation for corticosteroid-induced elevated intraocular pressure (Arch Soc Esp Oftalmol 2009; 84: 491-500).
\end{abstract}

\footnotetext{
Recibido: 12/8/08. Aceptado: 22/10/09.

Sección de Glaucoma del Servicio de Oftalmología del Hospital Ramón y Cajal.

1 Doctor en Medicina.

2 Licenciado en Medicina.

Correspondencia:

Francisco J. Muñoz Negrete

C/. Océano Antártico, 18

28760 Tres Cantos (Madrid)

España

E-mail: franciscojmunoz@telefonica.net
} 
ma primario de ángulo abierto. Finalmente, se está evaluando la mifepristona tópica, antagonista de receptores glucocorticoides, en la elevación de la presión ocular secundaria al uso de esteroides.

Palabras clave: Glaucoma, tratamiento médico glaucoma, prostaglandinas, combinaciones fijas.
Key words: Glaucoma, glaucoma medical treatment, prostaglandins, fixed combinations, calcium channel blockers.
La presión intraocular (PIO) elevada es uno de los principales factores de riesgo de desarrollo de Glaucoma. El manejo inicial consiste en disminuir la presión intraocular mediante el uso de fármacos. Desde que en 1870 la pilocarpina fue empleada para el tratamiento del glaucoma, una intensa actividad investigadora se centra en torno a esta patología con el fin de descubrir nuevos principios activos más potentes, con efectos más prolongados y mayor seguridad y tolerancia.

Las novedades actuales se centran en variaciones de principios activos preexistentes, que intentan mejorar la tolerancia local, fundamentalmente con la aparición de nuevos conservantes o formulaciones sin conservante y también en la descripción de nuevos grupos farmacológicos, algunos de ellos con mecanismos de acción diferentes a los actuales. A continuación pretendemos exponer una revisión de las novedades más destacadas que se están desarrollando en el tratamiento farmacológico del glaucoma (tabla I).

Tabla I. Productos antiglaucomatosos futuros en estudio

1) Análogos prostaglandinas

a) AR-102

b) $\mathrm{PF}-03187207$

2) Antagonistas del Calcio

a) Lomerizina

b) Flunarizina tópico

c) Iganidipina

3) Antagonistas de la endotelina

a) Sulfisoxazol

b) Bunazosina tópico

4) Agonistas/Antagonistas receptores Renina-Angiotensina

a) Olmesartan tópico

b) Angiotensina (1-7)

5) Inhibidores de la Rho-kinasa (ROCK)

a) Y-27632 tópico

6) Antagonistas de los receptores de los esteroides a) Mifepristona tópico

\section{AGONISTAS DE PROSTAGLANDINAS}

El uso de análogos de las prostaglandinas como hipotensores oculares en el tratamiento del glaucoma se propuso a principio de los años ochenta por Camras y Brito (1). Desde entonces los agonistas de los receptores FP de las prostaglandinas comercializados, latanoprost (Xalatan ${ }^{\circledR}$, Pfizer, S.A. Madrid, España), bimatoprost (Lumigan $^{\circledR}$, Allergan S.A., Tres Cantos, Madrid, España) y Travoprost (Trava$\tan ^{\circledR}$, Alcon Cusí, S.A., El Masnou, Barcelona, España) han demostrado una elevada potencia hipotensora ocular, superior a los beta-bloqueantes tópicos, tanto en pacientes con diagnóstico de glaucoma primario de ángulo abierto como en glaucoma de ángulo estrecho $(2,3)$. Si a esto sumamos la baja incidencia de complicaciones sistémicas, puede entenderse que sean considerados fármacos antiglaucomatosos de primera línea en el momento actual.

En los productos comercializados se está intentando mejorar su tolerancia con cambios en su formulación. Tal es el caso de Travatan- $\mathbf{Z}^{\circledR}$ (Alcon, Fort Worth, Texas, USA), en el que se sustituye el cloruro de Benzalkonio por sofZia, un conservante ionizado que presenta menor toxicidad sobre la superficie ocular sin restar eficacia hipotensora al preparado (4). Como curiosidad ha sido descrito un paciente que desarrolló edema macular cistoide tras cambio de tratamiento de latanoprost a Travatan-Z, postulando los autores que el efecto pudiera estar relacionado más con el conservante que con el producto activo (5).

Otras novedades que pronto se producirán en el mercado español son la aparición de análogos de las prostaglandinas genéricos. Aunque teóricamente deberían ser igualmente efectivos, recientemente ha sido publicado un estudio en la India en el que se observa que el preparado genérico de Latanoprost 
(Latoprost ${ }^{\circledR}$ ) era de menor eficacia hipotensora que Xalatan ${ }^{\circledR}$, siendo los efectos adversos locales similares. Este hecho tendrá que analizarse cuando se comercialicen análogos de prostaglandinas genéricos en nuestro país (6).

A la lista de análogos de prostaglandinas hoy en día presentes en el mercado, se unen otros nuevos que esperan aumentar la potencia farmacológica y disminuir la iatrogenia ocular minimizando el uso de conservantes. El Tafluprost (Saflutan ${ }^{\circledR}$, Merck Sharp \& Dohme, S.A., Madrid, España) es un agonista de receptores-FP prostanoides recientemente aprobado. Se trata de un éster de isopropil que en contacto con la superficie ocular es hidrolizado rápidamente por las esterasas corneales convirtiéndose en un metabolito clínicamente activo. Presenta gran afinidad por los receptores FP y en el estudio preclínico que se ha llevado a cabo parece que su efecto hipotensor es superior al del latanoprost en ratones y monos con tensión ocular normal (7). Asimismo su aplicación tópica en gatos ha mostrado un incremento en el flujo sanguineo retiniano superior al latanoprost que sugiere un efecto similar en pacientes afectos de glaucoma en los que podría mejorar la perfusión ocular (8).

Es sabido que los análogos de prostaglandinas causan pigmentación iridiana, crecimiento de las pestañas e hiperemia conjuntival. Si bien estos fenómenos son esperables con el uso del nuevo prostanoide, se ha observado que a diferencia del latanoprost, el tafluprost no incrementa la melanogénesis en las células melánicas cultivadas in vitro (9). Se precisan estudios clínicos a largo plazo para evaluar si el tafluprost produce menos hiperpigmentación de iris en humanos que el resto de análogos de prostaglandinas comercializados.

Por otra parte, la presentación de Tafluprost sin conservantes podría suponer una ventaja adicional en cuanto a toxicidad ocular comparado con los tradicionales análogos de prostaglandinas, que llevan asociado cloruro de benzalkonio (10). Sin embargo, Sutton et al encuentran, en un estudio controlado con placebo, una mayor incidencia de hiperemia conjuntival y fotofobia en el grupo de pacientes tratados con tafluprost frente al grupo que recibió Latanoprost (11).

Otros análogos de prostaglandinas en fase de investigación son AR-102 (Aerie Pharmaceuticals Inc) y PF-03187207 (Pfizer y NicOx) (12). Respecto a AR-102, los estudios preclínicos indican que podría ser ciento cincuenta veces más selecti- vo y treinta veces más potente para los receptores FP que latanoprost. Los estudios preclínicos son esperanzadores y hablan de mayor eficacia hipotensora, mayor duración del efecto que latanoprost y mejor tolerancia que travoprost. En cuanto al PF-03187207 es un análogo de prostaglandinas F2-alfa que asocia propiedades donadoras de óxido nítrico, sin embargo tras la realización de los estudios clínicos iniciales el producto no va a ser comercializado.

\section{ANTAGONISTAS DEL CALCIO}

El desarrollo de ciertas enfermedades del nervio óptico se ha relacionado con un flujo sanguíneo ocular insuficiente. En el caso del glaucoma, su desarrollo podría deberse en parte al compromiso de la circulación de la cabeza del nervio óptico asociado al aumento de la PIO. Los fármacos calcio-antagonistas, que son ampliamente empleados para el tratamiento de la hipertensión arterial y las enfermedades coronarias, reducen el tono de los vasos sanguíneos inhibiendo la entrada de calcio en las células, lo que causa una relajación del músculo liso y un aumento regional del flujo sanguíneo en diversos tejidos. Distintas investigaciones sugieren que el uso sistémico de calcio-antagonistas podría mejorar el pronóstico de al menos parte de los pacientes con glaucoma, al incrementar el flujo sanguíneo ocular y tener un efecto neuroprotector sobre las células ganglionares de la retina, dado que la hipoxia de las células ganglionares de la retina parece estar relacionada, al menos en parte, con el aumento de los niveles de calcio intracelular. Sin embargo, el uso de estos productos en el glaucoma es controvertido, ya que recientemente se ha publicado que el uso de antagonistas del calcio podría estar ligado a una mayor tasa de glaucoma primario de ángulo abierto, lo que iría en contra su uso, previamente recomendado por algunos autores, en el glaucoma primario de ángulo abierto (13).

La lomerizina es un calcioantagonista mixto que actúa tanto sobre los receptores T como L del calcio. Ha mostrado una mejoría selectiva del flujo sanguíneo retiniano y de la cabeza del nervio óptico tanto en conejos como humanos (14) y un efecto neuroprotector en conejos (15) y ratas (16), sin mostrar apenas efectos significativos sobres la presión sistémica y la frecuencia cardíaca (14). Puede 
considerarse por tanto, como un vasodilatador a nivel de los tejidos oculares sin efectos hipotensores sistémicos. Su aparente selectividad por las arterias cerebrales incrementa la esperanza de que, en el ojo, el efecto vascular de este fármaco sea más selectivo a nivel de los tejidos neurales (lo cual parece estar refrendado por el hecho de que la lomerizina aumenta el flujo sanguíneo retiniano y de la cabeza del nervio óptico sin modificar el flujo coroideo). La relativa falta de influencia sobre la presión sistémica podría hacer que la lomerizina fuera más idónea para uso oftálmico que el resto de calcioantagonistas, si bien puede también ocasionar efectos secundarios como la somnolencia o el rubor facial.

La flunarizina es un calcioantagonista mixto que, como la lomerizina, actúa sobre los receptores T y L del calcio, y a su vez actúa como antagonista de los canales de sodio. Su mecanismo de acción parece consistir en la inhibición de la captación de dopamina y la unión a los receptores de dopamina, principalmente los de tipo D2. Al igual que otros antagonistas de los receptores de DA, interactúa con los receptores sigma, presentes en el iris y cuerpo ciliar del ojo de conejo, relacionados con la producción y drenaje del humor acuoso. Su aplicación tópica parece disminuir la presión intraocular tanto en ojos de conejo normotensivos como en ojos con hipertensión inducida por alfa-quimotripsina (17) y en perros (18).

La Iganidipina es un nuevo derivado dihidropiridínico de los calcioantagonistas, relativamente soluble en agua y el único de sus características actualmente disponible como solución oftálmica (19). Su instilación tópica aumenta el flujo sanguíneo de la cabeza del nervio óptico ipsilateral en conejos y monos (20,21), e inhibe la contracción de los vasos sanguíneos inducida por la endotelina-1 (19).

En general, el uso de calcioantagonistas puede provocar la aparición de efectos secundarios derivados del bloqueo de los canales de calcio a nivel de las arterias periféricas como hipotensión, palpitaciones, taquicardia refleja, edemas periféricos y vértigo. Actualmente existe evidencia de que el efecto de la lomerizina a nivel de la presión sanguínea es menor que en el resto de calcioantagonistas, debido a que ésta inhibe selectivamente la entrada de calcio dependiente de canales de $\mathrm{Ca}++/ \mathrm{KCl}$ más potentemente a nivel de las arterias cerebrales que de las mesentéricas.

\section{ANTAGONISTAS DE LA ENDOTELINA}

Las endotelinas son una familia de isopéptidos (ET1, ET2 y ET3) codificados por diversos genes localizados en distintos loci. Las ET1 y ET2 actúan como potentes vasoconstrictores, a diferencia de la ET3 que actúa como un débil vasoconstrictor en comparación con las anteriores. Además de los efectos vasoactivos producen múltiples efectos en tejidos no vasculares, incluyendo el tejido nervioso. Actúan a nivel de los receptores ETa y ETb $(22,23)$. De éstos, el receptor ETa presenta una afinidad selectiva por la ET1 mientras que el receptor ETb presenta la misma afinidad por las tres isoformas. El receptor ETa está principalmente asociado con el músculo liso de los vasos sanguíneos (24) y su activación produce vasoconstricción, mientras que el receptor ETb es más abundante en los astrocitos (25).

Las endotelinas han mostrado jugar un papel importante en la homeostasis tanto de la cámara anterior como posterior del ojo. En el segmento anterior, se han detectado elevados niveles de ET1 y ET3 a nivel de iris, cuerpo ciliar y humor acuoso, mientras que los receptores Eta y ETb han sido localizados a nivel de iris, músculo ciliar y procesos ciliares en ratas, conejos y humanos. Parece ser que la ET1 juega su papel en la regulación de la PIO (26). En el segmento posterior, se ha detectado ET1 y ET3 inmmunorreactivas y su ARNm a nivel de coroides y retina.

Además de intervenir en la homeostasis del ojo, las endotelinas podrían jugar cierto papel en la patogénesis del glaucoma (27). Así, se han observado niveles elevados de ET1 en el humor acuoso y el plasma de humanos, perros y ratas con glaucoma.

Estudios recientes intentan evaluar la capacidad de los antagonistas de los receptores de las endotelinas para actuar como neuroprotectores, barajándose distintos mecanismos de acción. A nivel vascular, en situaciones de isquemia se produce la liberación de ET1 que causa vasoconstricción en la cabeza del nervio óptico, que podría ser seguida de isquemia y muerte celular; a esto hay que añadir que en situaciones de aumento de PIO también aumentan los niveles de ET1 en la cabeza del nervio óptico (28). Así pues los antagonistas de la endotelina protegerían a las neuronas previniendo la hipoperfusión post-isquémica de la retina y la vasoconstricción de la cabeza del nervio óptico. Por otro lado, a nivel no vascular, en condiciones de isque- 
mia, los astrocitos quiescentes del cerebro, retina y cabeza del nervio óptico sufren un proceso de astrogliosis por el cual se produce la liberación de mediadores tóxicos y la muerte neuronal (29). En este caso, los antagonistas de la endotelina también actuarían inhibiendo esta cascada.

El sulfisoxazol es un antagonista no selectivo de la endotelina. Estudios experimentales en ratas han demostrado recientemente que su uso protege la retina frente al daño isquémico en procesos como el glaucoma, reduciendo las alteraciones del electrorretinograma y normalizando los cambios en los niveles de acetilcolintransferasa, óxido nítrico sintetasa, Thy-1(marcador específico de las células ganglionares de la retina) y FGF-2 (factor trófico producido por las células gliales en situaciones de estrés), propios de las situaciones de isquemia (30).

La bunazosina es un potente antagonista selectivo de los adrenoreceptores alfa 1 . Se ha observado que tras su instilación en ojos de conejo normotensivos, penetra localmente y alcanza la retina a concentraciones suficientes para atenuar la vasoconstricción de las arterias retinianas inducida por fenilefrina o endotelina-1. En humanos adultos, la aplicación tópica de bunazosina incrementa la velocidad de flujo sanguíneo en nervio óptico, retina y coroides, sin alterar la tensión arterial ni la frecuencia cardiaca. Estas cualidades podrían determinar que fuera útil en enfermedades retinianas isquémicas y en glaucomas con componente vascular (31).

\section{AGONISTAS/ANTAGONISTAS DE LOS RECEPTORES DE ANGIOTENSINA}

Es ampliamente conocido el papel que juega el sistema renina-angiotensina (RAS) en el control de la presión arterial sistémica y la homeostasis iónica del medio interno. Recientemente se ha demostrado que la angiotensina II no sólo tiene una potente acción vasoconstrictora y estimulante de la liberación de aldosterona sino que actúa además como factor de crecimiento e inmunomodulador en fenómenos de proliferación, fibrosis y apoptosis celular.

$\mathrm{Si}$ bien es conocida su acción en tejidos tales como el adrenal, renal y vascular, muchos de los componentes del sistema RAS se han identificado en ojos humanos y animales. Concretamente son expresados en el cuerpo ciliar, tejido responsable de la producción de humor acuoso. Su presencia y relevancia en la malla trabecular es objeto de estudio, ya que autores como Shen et al (32) han evidenciado un papel pro-proliferativo y pro-fibrótico de la Angiotensina II a dicho nivel en modelos animales. Sin embargo, y a diferencia de lo que cabría esperar de estos hallazgos, Yan Ke at al no encontraron diferencias estadísticamente significativas en los niveles de Angiotensina II circulante en pacientes con glaucoma normotensivo comparado con controles (33).

Al igual que la Angiotensina I y II no son capaces de atravesar la barrera hematoencefálica, tampoco pueden alcanzar el vítreo en caso de que la barrera hemato-retiniana esté intacta (34). Por ello se han desarrollado en los últimos años distintos estudios para evaluar la acción del RAS en el sistema ocular.

La aplicación tópica de enzima convertidor de la angiotensina mostró ya a finales de los años ochenta una disminución de la PIO en pacientes con hipertensión ocular y glaucoma primario de ángulo abierto (35).

Tras evidenciarse la influencia de la Angiotensina II sobre el drenaje uveo-escleral mediado por los receptores AT1 (36), Wang et al (37) emplean olmesartan (CS-088), un antagonista de los receptores de angiotensina II tipo AT1, de forma tópica en monos con glaucoma unilateral inducido por láser observando una reducción de la PIO dosis-dependiente.

Un nuevo metabolito del RAS, la angiotensina (1-7), producto de la acción de carboxi y endopeptidasas sobre dos formas de angiotensina, I y II, ha demostrado tener efectos contrarios a los de su precursor, actuando como factor vasodilatador y antiproliferativo biológicamente activo (38-41). Parece ser que estas acciones serían mediadas por un receptor Mas, ligado a la proteína $\mathrm{G}$, exclusivo de Angiotensina (1-7) ligando y sin actividad frente a la angiotensina II.

En un estudio recientemente publicado (42), se ha evaluado el efecto de la angiotensina II y su metabolito Angiotensina (1-7) en la PIO intraocular y cambios en el flujo de humor acuoso empleando un modelo animal con normotensión ocular. Se observó como la aplicación tanto tópica como intravítrea de Angiotensina II se acompañó de un aumento significativo de la resistencia a la salida de humor acuoso sin que ello supusiera un cambio en la PIO; efecto que se revertía con una antagonista AT1 como el olmesartan. Por otra parte, la angiotensina (1-7) redujo la PIO, probablemente vía 
receptores Mas, sin que se registraran cambios a nivel del flujo de salida de humor acuoso. Ello sugiere que posiblemente su mecanismo de acción mediado por los receptores Mas esté relacionado más con la inhibición de la producción que con el aumento de drenaje de humor acuoso.

\section{INHIBIDORES DE LA RHO-KINASA (ROCK)}

En la mayoría de las formas de glaucoma la elevación de la PIO parece ser el resultado de una dificultad en el drenaje del humor acuoso a nivel de la malla trabecular y el canal de Schlemm. Son varios los estudios que han tratado de identificar el mecanismo patogénico real por el cuál cambios en la malla trabecular modulan la filtración del humor acuoso y qué fármacos podrían modificar su comportamiento. La malla trabecular posee propiedades similares a las del músculo liso y es posible que sea su capacidad contráctil la que permita regular la filtración de humor acuoso a su través ya sea por cambios en el tamaño de los espacios de drenaje de la malla, en la permeabilidad del canal de Schlemm o en la matriz extracelular.

La Rho guanosin trifosfatasa (Rho GTPasa) es una proteína transmembrana de la Superfamilia Ras que a través de su efector, la proteína Rho Kinasa (ROCK), controla actividades celulares tales como la contracción muscular, la organización del citoesqueleto, la adhesión celular o la migración de células de la malla trabecular cultivadas in vitro.

Ponugoti et al han observado que la inhibición del enzima Rho-Kinasa produce cambios en la morfología de las células trabeculares, en las uniones intercelulares e inhibe la fosforilación de las cadenas ligeras de miosina, lo que se correlaciona con una mejoría en la salida de humor acuoso (43).

Posteriormente, Koga et al (44) estudiaron el comportamiento de células humanas de la malla trabecular cultivadas in vitro tratadas con el Y-27632, un inhibidor selectivo de la ROCK. Los hallazgos fueron en parte contradictorios con lo que otros autores, incluidos ellos mismos, habían encontrado previamente: los inhibidores de la Rho-Kinasa inducían cambios en la morfología celular, aumentando la adherencia celular, las proyecciones citoplasmáticas y acelerando la migración celular. Así pues, la reducción de la PIO asociada al tratamiento tópico con Y- 27632 descrito por Inatani et al
(45) y documentada previamente en estudios animales, podría explicarse por estos cambios inducidos en la actividad celular de la malla trabecular.

Siguiendo esta línea de investigación y alentados por la ausencia de toxicidad celular asociada al Y27632 en los estudios previos, Rao et al (46) ponen de manifiesto otras propiedades de los inhibidores de la ROCK, tales como el aumento del flujo sanguíneo ocular, la supervivencia de las células ganglionares retinianas y la regeneración axonal.

Honjo et al (47) han investigado su capacidad de regular la actividad fibroblástica en la cápsula de Tenon tras cirugía penetrante de glaucoma en conejos. Encontraron que la instilación postoperatoria tópica de este producto tenía efectos favorables en la prevención de la fibrosis de la ampolla de filtración.

Por tanto, los inhibidores de la ROCK podrían actuar en el tratamiento del glaucoma por un mecanismo de acción nuevo, que podríamos denominar «trabecular» y que se dirigiría más directamente a la patogenia real del glaucoma que los fármacos que utilizamos en la actualidad.

\section{ANTAGONISTAS DE LOS RECEPTORES DE ESTEROIDES}

La mifepristona es un antagonista específico de los receptores glucocorticoides que, aplicada vía tópica en conejos, produce una disminución modesta de la PIO.

En estudios experimentales se observó que animales tratados con mifepristona y a continuación con dexametasona no mostraron un incremento de la PIO (48).

También se han realizado estudios acerca de la posible interacción entre la progesterona y la mifepristona y su repercusión sobre la PIO en ojos de conejo. Se ha visto que la administración aislada de medrisona (progesterona) aumenta significativamente la PIO durante las tres primeras semanas, mientras que cuando la medrisona es administrada simultáneamente con la mifepristona, la PIO aumenta inicialmente pero a las 2 semanas vuelve a sus niveles iniciales. Los niveles de PIO también disminuyen cuando la mifepristona es administrada 2 semanas después de la medrisona (49). Así, parece que el uso de la mifepristona consigue antagonizar los efectos de la progesterona sobre la presión intraocular. 
Una formulación de mifepristona tópica (RU486) se encuentra en estudios en fase 2 como tratamiento de la elevación de la PIO secundaria al uso de corticoides (12).

\section{FÁRMACOS NEUROPROTECTORES}

La memantina oral, aprobada para uso en la enfermedad de Alzheimer ha sido estudiada como agente neuroprotector en glaucoma, sin embargo finalmente no ha sido aprobado su uso en glaucoma (12). También la casa Neurotech está investigando el uso de implantes intravítreos para liberación sostenida de factores de crecimiento al polo posterior (12).

\section{COMBINACIONES FIJAS}

Todas las combinaciones fijas de fármacos antiglaucomatosos comercializadas contienen maleato de timolol 0,5\%, que se ha combinado con análogos de prostaglandinas, como latanoprost (Xalacom ${ }^{\circledR}$, Pfizer, Madrid, España), travoprost (Duotrav $^{\circledR}$, Alcon Cusí, S.A., El Masnou, Barcelona, España) y bimatoprost $\left(\right.$ Ganfort $^{\circledR}$, Allergan S.A., Tres Cantos, Madrid, España), con alfa2agonistas como la brimonidina (Combigan ${ }^{\circledR}$, Allergan S.A., Tres Cantos, Madrid, España) y con inhibidores de la anhidrasa carbónica tópicos como la dorzolamida $\left(\right.$ Cosopt $^{\circledR}$, Merck Sharp \& Dohme, S.A., Madrid, España) y la brinzolamida (Azarga ${ }^{\circledR}$, Alcon Cusí, S.A., El Masnou, Barcelona, España), que ha sido el último en comercializarse en España. Un estudio randomizado incluyendo más de 500 pacientes ha demostrado que esta combinación es más eficaz que cada uno de los componentes de la misma. No se han referido efectos adversos a nivel cardiovascular, aunque este hecho está sesgado por los criterios de selección de la muestra que no permiten incluir a pacientes con contraindicaciones a los betabloqueantes (50). En un reciente estudio comparativo de las dos combinaciones fijas que contienen inhibidores de la anhidrasa carbónica, se ha observado una eficacia comparable. Respecto a los efectos adversos, se han encontrado más molestias e irritación ocular con la combinación fija que contiene dorzolamida y más visión borrosa con la que contiene brinzolamida, al igual que sucedía previa- mente con estos dos componentes aplicados de forma aislada sin betabloqueante (51).

En México se ha comercializado una combinación fija con tres productos activos: timolol, brimonidina y dorzolamida (Krytantek Ofteno ${ }^{\circledR}$, Laboratorios Sophia SA de CV, Zapopan, México). Los resultados iniciales indican que presenta una mayor eficacia que las combinaciones fijas timolol-dorzolamida y timolol-brimonidina (Baiza-Durán LM, Varma R, Krytantek Ofteno Study Group ARVO).

\section{BIBLIOGRAFÍA}

1. Camras CB, Bito LZ, Eakins KE. Reduction of intraocular pressure by prostaglandins applied topically to the eyes of conscious rabbits. Invest Ophthalmol Vis Sci 1977; 16: 1125-1134.

2. Zhang WY, Po AL, Dua HS, Azuara-Blanco A. Meta-analysis of randomised controlled trials comparing latanoprost with timolol in the treatment of patients with open angle glaucoma or ocular hypertension. Br J Ophthalmol. 2001; 85: 983-990.

3. Chew PT, Aung T, Aquino MV, Rojanapongpun P; EXACT Study Group. Intraocular pressure-reducing effects and safety of latanoprost versus timolol in patients with chronic angle-closure glaucoma. Ophthalmology 2004; 111: 427-434.

4. Brasnu E, Brignole-Baudouin F, Riancho L, Guenoun JM, Warnet JM, Baudouin C. In Vitro effects of preservativefree tafluprost and preserved latanoprost, travoprost and bimatoprost in a conjunctival epithelial cell line. Curr Eye Res 2008; 33: 303-312.

5. Esquenazi S. Cystoid macular edema in a pseudophakic patient after switching from latanoprost to BAK-free travoprost. J Ocul Pharmacol Ther 2007; 23: 567-570.

6. Narayanaswamy A, Neog A, Baskaran M, George R, Lingam V, Desai $C$, et al. A randomized, crossover, open label pilot study to evaluate the efficacy and safety of Xalatan in comparison with generic Latanoprost (Latoprost) in subjects with primary open angle glaucoma or ocular hypertension. Indian J Ophthalmol 2007; 55: 127-131.

7. Ota T, Murata H, Sugimoto E, Aihara M, Araie M. Prostaglandin analogues and mouse intraocular pressure: Effects of tafluprost, latanoprost, travoprost, and unoprostone considering 24-hour variation. Invest Ophthalmol Vis Sci 2005; 46: 2006-2011.

8. Takagi Y, Nakajima T, Shimazaki A, Kageyama M, Matsugi T, Matsumura Y, et al. Pharmacological characteristics of AFP-168 (tafluprost), a new prostanoid FP receptor agonist, as an ocular hypotensive drug. Exp Eye Res 2004; 78: 767-776.

9. Nakajima T, Matsugi T, Goto W, Kageyama M, Mori N, Matsumura $Y$, et al. New fluoroprostaglandin F2. derivatives with prostanoid FP-receptor agonistic activity as potent ocular hypotensive agents. Biol Pharma Bull 2003; 26: 1691-1695.

10. Sutton A, Gikvarry A, Ropo A. A comparative,placebocontrolled study of prostanoid fluoroprostaglandin recep- 
tor agonists tafluprost and latanoprost in healthy males. $J$ Ocul Pharmacol Ther 2007; 23: 359-365.

11. Gross RL, Peace JH, Smith SE, Walters TR, Dubiner HB, Weiss MJ, et al. Duration of IOP reduction with travoprost BAK-free solution. J Glaucoma 2008; 17: 217-222.

12. Novack GD. Update on new pharmaceutical products. In: Coleman AI, Jampel HD. Glaucoma 2007: Achieving balance. San Francisco: American Academy of Ophthalmology 2007: 35-36.

13. Müskens RP, de Voogd S, Wolfs RC, Witteman JC, Hofman A, de Jong PT, et al. Systemic Antihypertensive Medication and Incident Open-angle Glaucoma. Ophthalmology 2007; 114: 2221-2226.

14. Tamaki Y, Araie M, Fukaya Y, Nagahara M, imamura A, Honda $M$, et al. Effects of Lomerizine, a calcium channel antagonist, on retinal and optic nerve head circulation in rabbits and humans. Invest Ophthalmol Vis Sci 2003; 44: 4864-4871.

15. Danielisova V, Chavko M. KB-2796, a calcium channel blocher, ameliorates ischemic spinal cord damage in rabbits. Neurochem Res 1994: 19: 1503-1507.

16. Yamashita A, Ozaki A, Ikegami A, Hayashi A, Hara H, Sukamoto T, et al. Effects of a new diphnylpiperazine calcium antagonist, $K B-2796$, on cerebral ischemic neuronal damage in rats. Gen Pharmacol 1993; 24: 1473-1480.

17. Campana $G$, Bucolo $C$, Murari G, Spampinato $S$. Ocular hypotensive action of topical flunarizine in the rabbit. Role of sigma recognition sites. J Parmacol Exp Ther 2002; 303: 1086-1094.

18. Greller AL, Hoffman Ar, Liu C, Ying GS, Vudathala DK, Acland GM, et al. Effects of the topically applied calciumchannel blocker flunarizine on intraocular pressure in clinically normal dogs. Am J Vet Res 2008; 69: 273-278.

19. Ishii K, Matsuo H, Fukaya Y, Tanaka S, Sakaki H, Waki M, et al. Iganidipine, a new water-soluble Ca 2+ antagonist:ocular and periocular penetration after instillation. Invest Ophthalmol Vis Sci 2003; 44: 1169-1177.

20. Ishii K, Fukaya Y, Araie M, Tomita G. Topical administration of iganidipine, a new water-soluble Ca 2+ antagonist, increases ipsilateral optic nerve head circulation in rabbits and cynomolgus monkeys. Curr Eye Res 2004; 29: 67-73.

21. Waki. M, Sugiyama T, Watanabe N, Ogawa T, Shirahase H, Azuma I. Effect of topicaly applied iganidipine dihydrochloride, a novel Ca 2+ antagonist, on optic nerve head circulation in rabbits. Nippon Ganka Gakkai Zasshi 2000; 104: 541-546.

22. Arai H, Hori S, Aramori I, Onkubo H, Nakanishi S. Cloning and expression of a cDNA encoding an endothelin receptor. Nature 1990; 348: 730-732.

23. Sakurai T, Yanagisawa M, Masaki T. Molecular characterization of endothelin receptors. Trends Pharmacol Sci 1992; 13: 103-108.

24. Pierre LN, Davenport AP. Relative contribution of endothelin $A$ and endothelin $B$ receptors to vasoconstriction in small arteries from human heart and brain. J Cardiovas Pharmacol 1998; 31: S74-S76.

25. Schinelli S, Zanassi P, Paolillo M, Wang H, Feliciello A, Gallo $V$. Stimulation of endothelin B-receptors in astrocytes induces cAMP response element-binding protein phosphorylation and c-fos expression via multiple mitogenactivated protein kinase signalling pathways. J Neurosci 2001; 21: 8842-8853.
26. Wiederholt M. Direct involvement of trabecular meshwork in the regulation of aqueous humor outflow. Curr Opin Ophthalmol 1998; 9: 46-99.

27. Yorio T, Krishnamoorthy R. Prasanna G. Endothelin: is it a contributor to glaucoma pathophysiology? J Glaucoma 2002; 11: 259-270.

28. Prasanna G, Hulet $C$, Desai D, Krishnamoorthy $R R$, Narayan S, Brun AM, et al. Effect of elevated intraocular pressure on endohelin-1 in a rat model of glaucoma. Pharmacol Res 2005; 51: 41-50.

29. Tacconi MT. Neuronal death: is there a role for astrocytes?. Neurochem Res 1998; 23: 759-765.

30. Syed H, Safa R, Chidlow G, Osborne NN. Sulfisoxazole, an endothelin receptor antagonist, protects retinal neurones from insults of ischemia/reperfusion or lipopolysaccharide. Neurochem Int 2006;48: 708-717.

31. Hara H, Ichikawa M, Oku H, Shimazawa M, Araie M. Bunazosin, a selective alphal-adrenoceptor antagonist, as an anti-glaucoma drug: effects on ocular circulation and retinal neuronal damage. Cardiovasc Drug Rev 2005; 23: 43-56.

32. Shen F, Zhang L, Liu T. Effects of angiotensin II on the $3 H$ $T d R$ incorporation and synthesis of collagen in cultured bovine trabecular meshwork cells. 2001; 17: 209-212.

33. Yun $H, F u P$, Ding $K$, Yang $Q$. The comparison study of renin angiotensin A II levels on normal tension glaucoma patients and normal individuals. Yan Ke Xue Bao 2005; 21: 192-195.

34. Danser AH, Derkx FH, Admiraal PJ, Deinum J, de Jong PT, Schalekamp MA. Angiotensin levels in the eye. Invest Ophthalmol Vis Sci 1994; 35: 1008-1018.

35. Constad WH, Fiore P, Samson C, Cinotti AA. Use of an angiotensin converting enzyme inhibitor in ocular hypertension and primary open-angle glaucoma. Am J Ophthalmol 1988; 105: 674-677.

36. Inoue T, Yokoyoma T, Koike H. The effect of angiotensin II on uveoscleral outflow in rabbits. Curr Eye Res 2001; 23: 139-143.

37. Wang RF, Podos SM, Mittag TW, Yokoyoma T. Effect of CS088, an angiotensin AT1 receptor antagonist, on intraocular pressure in glaucomatous monkey eyes. Exp Eye Res 2005; 80: 629-632.

38. Ueda S, Masumori-Maemoto S, Wada A, Ishii M, Broshinan KB, Umemura S. Angiotensin (1-7) potentiates bradykinin-induced vasodilatation in man. J Hypertens 2001; 19: 2001-2009.

39. Santos RA, Simoes e Silva AC, Maric C, et al. Angiotensin (1-7) is an endogenous ligand for the G-protein-coupled receptor Mas. Proc Natl Acad Sci USA 2003; 8: 8258-8263.

40. Iwata M, Cowling RT, Gurantz D, Moore C, Zhang S, Yuan $J X$, et al. Angiotensin (1-7) binds to specific receptors on cardiac fibroblasts to initiate antifibrotic and antitrophic effects. Am J Physiol Heart Circ Physiol 2005; 289: H2356-2363.

41. Kostenis E, Milligan G, Christopoulos A, Sanchez-Ferrer $C F$, Heringer-Walther S, Sexton PM, et al. G-protein-coupled Receptor Mas is a physiological Antagonist of the Angiotensin II type I receptor. Circulation 2005; 111: 1806-1813.

42. Vaajanen AE, Vapaatalo HI, Kautiainen $H$, Oksala $O$. Angiotensin (1-7) reduces intraocular pressure in the normotensive rabbit eye. Invest Ophthalmol Vis Sci 2008; 49: 2557-2562. 
43. Rao PV, Deng P, Maddala R, Epstein DL, Li CY, Shimokawa $H$. Expression of dominant negative Rho-binding domain of Rho-Kinase in organ cultured human eye anterior segments increases aqueous humor outflow. Mol Vis 2005; 11: 288-297.

44. Koga T, Koga T, Awai M, Tsutsui J, Yue B, Tanihara H. Rho-associated protein kinase inhibitor, $Y$ - 27632, induces alterations in adhesion, contraction and motility in cultured human trabecular meshwork cells. Exp Eye Res 2006; 82: $362-370$.

45. Inatani $M$, Honjo $M$, Tokushige $H$, Azuma J, Araie M. Intraocular Pressure-Lowering effects and safety of topical administration of a Selective ROCK inhibitor, SNJ-1656, in healthy volunteers. Arch Ophthalmol 2008; 126: 309-315.

46. Rao VP, Epstein DL. Rho GTPase/Rho Kinase inhibition as a novel target for the treatment of glaucoma. BioDrugs 2007; 21: 167-177.

47. Honjo M, Tanihara H, Kameda T, Kawaji T, Yoshimura N, Areie M. Potencial role of Rho-associated protein Kinase inhibitor Y-27632 in glaucoma filtration surgery. Invest Ophthalmol Vis Sci 2007; 48: 5549-5557.

48. Green K, Cheeks L, Slagle T, Phillips CI. Effects of mifepristone on rabbit intraocular pressure in the presence and absence of dexamethasone. Ophthalmic Res 1990; 22: 247-252.

49. Green $K$, Cheeks L, Slagle T, Phillips CI. Interaction between progesterone and mifepristone on intraocular pressure in rabbits. Curr Eye Res 1989; 8: 317-320.

50. Kaback M, Scoper SV, Arzeno G, James JE, Hua SY, Salem $C$, et al. Intraocular Pressure-Lowering Efficacy of Brinzolamide 1\%/Timolol 0.5\% Fixed Combination Compared with Brinzolamide 1\% and Timolol 0.5\%. Ophthalmology 2008; 115: 1728-1734.

51. Manni G, Denis P, Chew P, Sharpe ED, Orengo-Nania S, Coote MA, et al. The safety and efficacy of brinzolamide $1 \% /$ timolol $0.5 \%$ fixed combination vs dorzolamide $2 \% /$ timolol $0.5 \%$ in patients with open-angle glaucoma or ocular hypertension. J Glaucoma 2009; 18: 293-300. 